\title{
Implicancia de la lingüística textual en la comprensión lectora y nivel de logro, Perú
}

\author{
Implication of textual linguistics in reading comprehension and level of achievement, Peru \\ Implicação da linguística textual na compreensão da leitura e nível de desempenho, Peru
}

Jesús Manuel More López ${ }^{1}$

jmorel@unasam.edu.pe

https://orcid.org/0000-0003-0315-9810

\section{José Yovera Saldarriaga ${ }^{1}$}

joyo_sal@hotmail.com

https://orcid.org/0000-0001-5235-0270
Marco Antonio Jamanca Ramirez
majaramz@gmail.com
https://orcid.org/0000-0003-3779-5639

\author{
Dante Daniel Cruz Nieto ${ }^{2}$ \\ daniel2262@hotmail.com \\ https://orcid.org/0000-0003-0052-5619
}

${ }^{1}$ Universidad Nacional Santiago Antúnez de Mayolo, Huaraz, Perú

${ }^{2}$ Universidad Nacional José Faustino Sánchez Carrión, Huaura, Perú

Artículo recibido 23 de noviembre 2021, arbitrado y aceptado 17 de diciembre 2021 y publicado 7 de marzo 2022

\section{RESUMEN}

Para mejorar la comprensión lectora es importante aplicar unabuenalingüística textual. El objetivo del estudio radica en demostrar la importancia del uso en comprensión lectora en nivel logros. La metodología empleada fue bajo la investigación explicativa de diseño cuasi experimental, se utilizaron instrumentos escala de Likert y cuestionario. La población fue nivel secundario del I.E. No 20503 "José Carlos Mariátegui". Los resultados demostraron rechazar la hipótesis nula y aceptar hipótesis alterna al confirmarse que existen diferencias significativas después de la aplicación en las variables, con un nivel de confianza del 95\%; siendo la asintótica (bilateral) 0,000; la prueba de normalidad muestra que en ambos grupos las variables no se distribuyen según una ley normal; ya que la significancia asintótica (bilateral) de ambos grupos es 0,013 ; es decir para la comprensión lectora está por debajo del nivel de significación alfa prefijado $(0,05)$. Se concluye que, es interactiva, dinámica, interdisciplinaria en la comprensión y producción de textos.

Palabras clave: Lingüística textual; Comprensión; Lectura; Logros; Niveles

\section{ABSTRACT}

To improve reading comprehension it is important to apply good textual linguistics. The objective of the study is to demonstrate the importance of the use of reading comprehension at the achievement level. The methodology used was under the explanatory research of quasi-experimental design, Likert scale and questionnaire instruments were used. The population was secondary level of the I.E. No 20503 "José Carlos Mariátegui". The results showed that the null hypothesis was rejected and the alternative hypothesis was accepted when it was confirmed that there are significant differences after the application of the variables, with a confidence level of 95\%; being the asymptotic (bilateral) 0.000; the normality test shows that in both groups the variables are not distributed according to a normal law; since the asymptotic significance (bilateral) of both groups is 0.013 ; that is, for reading comprehension it is below the pre-set alpha significance level (0.05). It is concluded that, it is interactive, dynamic, interdisciplinary in the comprehension and production of texts.

Keywords: Textuallinguistics; Comprehension; Reading; Achievement; Levels

\section{RESUMO}

Para melhorar a compreensão de leitura, é importante aplicar uma boa lingüística textual. O objetivo do estudo é demonstrar a importância do uso da lingüística textual na compreensão da leitura no nível de realização. A metodologia utilizada estava sob a pesquisa explicativa do projeto quaseexperimental, foram utilizados instrumentos de escala Likert e questionário. A população era de nível secundário do I.E. No 20503 "José Carlos Mariátegui”. Os resultados mostraram que a hipótese nula foi rejeitada e a hipótese alternativa foi aceita quando foi confirmado que existem diferenças significativas após a aplicação das variáveis, com um nível de confiança de 95\%; sendo a assimptótica (bilateral) de 0,000; o teste de normalidade mostra que em ambos os grupos as variáveis não estão distribuídas de acordo com uma lei normal; já que a significância assimptótica (bilateral) de ambos os grupos é de 0,013; ou seja, para compreensão de leitura está abaixo do nível de significância alfa pré-estabelecido $(0,05)$. Conclui-se que, é interativo, dinâmico, interdisciplinar na compreensão e produção de textos.

Palavras-chave: Linguística textual; Compreensão; Leitura; Realização; Níveis; Compreensão da leitura 


\section{INTRODUCCIÓN}

En la actualidad los docentes del área de comunicación de la provincia de Barranca, emplean en su labor enfoques lingüísticos tradicionales; sin embargo, hay nuevos conocimientos que es necesario aplicarlos. Por lo que, esta situación evidencia que el Perú está ubicado en los últimos lugares en compresión lectora. Motivo por el cual, ha generado la preocupación por parte de los docentes. Esta afirmación se sostiene con Pisa (2009), que menciona la gran parte tiene dificultad en leer, comprender, analizar e interpretar los códigos de un texto. Esto se da entender que no todos tienen cualidades para ello; más aún cuando piden comprender un texto.

Debido a esta situación el docente se encuentra con dificultad, cuando sus alumnos no aprenden el mensaje del texto; creando múltiples vacíos en su labor, depende del grado de atención que muestre el estudiante y la forma cómo el docente explica. Por la cual, se debe mejorar en la comprensión del texto mediante la aplicación de estrategias metacognitivas en la planificación, supervisión y evaluación de la lectura; es decir en las fases que se desarrolló la lectura, planteados Muñoz, y Ocoña, 2017).

Es por eso que, se hace dificultoso realizar las críticas o juzgar las opiniones, lo cual se debe de tener en cuenta el enfoque de la lingüística textual desarrollando habilidades cognoscitivas, expresión e interpretación de los códigos textuales. Además, la teoría de la lingüística textual permite comprender textos literarios, también de manera sistemática y se convierte en una estrategia para la comprensión de textos (Gonzales, 2010). Por lo que, se evidenció el nivel de logro de los estudiantes que es bajo y en ello incide el necesario dominio de la interpretación de textos orales y escritos, la selección de textos (Santos,
2020). También se debe tener en cuenta que en las sesiones de aprendizajes tienen que ser óptimas las explicaciones, los argumentos, la creatividad e innovación según los autores (Mamani, et. al., 2021).

Asimismo, se evidenció que un texto es dotado de factores de textualización, lo que precisan ser observados para que todo significado tenga coherencia en las ideas. Se notó, también, que los aspectos textuales sustentan una producción de texto, en función a una determinada producción de texto en la que mencionan (Silva, y De Paiva, 2017). Por otro lado, la dimensión del texto es la argumentación dependiente de las relaciones de la coherencia. En donde la argumentación es constitutiva del discurso, pero es en el texto que se expresa citado por (Cavalcante, 2016).

Cabe mencionar, que el estudiante logra totalmente la adecuación en muy pocos aspectos de las propiedades de la lingüística textual, tales como: a) selecciona el lenguaje para producir el escrito, b) evita enunciados contradictorios, c) mantiene el referente por procedimientos gramaticales (Albarrán, 2015). Por lo consiguiente, las condiciones de producción del discurso, caracterizándose así, de manera multidisciplinaria y proporcionando al lector diferentes perspectivas sobre las formas de construir los sentidos al texto explicado por (Souza, 2017).

Por este motivo, se realizó la investigación sobre la implicancia de la lingüística textual en la comprensión lectora y nivel de logro, lo cual tuvo como objetivo demostrar la importancia del uso en la comprensión lectora en nivel logros. Lo cual tiene el propósito de mejorar el aprendizaje en los estudiantes, sean dinámicos y fomenten una comunicación horizontal que permita a los alumnos 
participar en clase y que despejen sus dudas ante de entender o comprender (Espinoza, et. al., 2021).

\section{MÉTODO}

El método empleado en la investigación es cuasi experimental; en la medida que el propósito de la misma fue demostrar relaciones de causa- efecto de las variables. Así mismo, consiste en organizar las condiciones de acuerdo en un plan previo con el fin de investigar relaciones causa-efecto; exponiendo a uno o más grupos experimentales, a la acción de una variable experimental y contrastan sus resultados con el grupo de control o de comparación citados por (Sánchez y Reyes, 1996).

En la investigación se usó el método explicativo porque permitió demostrar los efectos positivos, en las mediciones acerca de las variables. El diseño de la investigación fue de corte prospectivo, dado que la información se obtuvo siguiendo un procedimiento, teniendo en cuenta los objetivos de la investigación y después que se haya desarrollado la planificación del proyecto. También es de tipo longitudinal, dado que se midió los efectos positivos que se utilizaron instrumentos para ambas variables, considerando que están diseñados para aplicarlo tipo encuesta, por lo que se hizo antes y después en varios momentos y lugar. Según Gómez, (2006) menciona que bajo la perspectiva cuantitativa, la recolección de datos es equivalente a medir.

La población estuvo constituida por 1009 estudiantes, cuyas edades fluctuaron entre 6 a 17 años, comprendida entre los dos niveles: educación primaria básica con 469; secundaria con 540 alumnos de la Institución Educativa No 20503 “José Carlos Mariátegui”- Paramonga.
La muestra es finita, ya que la proporción es conocida, entonces se procesó con la siguiente fórmula:

$$
n=\frac{N \sigma^{2} Z^{2}}{e^{2}(N-1)+\sigma^{2} Z^{2}}
$$

Dónde:

$\mathrm{n}=$ el tamaño de la muestra.

$\mathrm{N}=$ tamaño de la población.

$a=$ Desviación estándar de la población que, generalmente cuando no se tiene su valor, suele utilizarse un valor constante de 0,5.

$\mathrm{Z}=$ Valor obtenido mediante niveles de confianza. Es un valor constante que, si no se tiene su valor, se lo toma en relación al 95\% de confianza equivale a 1,96 (como más usual) o en relación al 99\% de confianza equivale 2,58 , valor que queda a criterio del investigador.

e = Límite aceptable de error muestral que, generalmente cuando no se tiene su valor, suele utilizarse un valor que varía entre el $1 \%$ $(0,01)$ y $9 \%(0,09)$, valor que queda a criterio del encuestador.

Ordenando se obtiene la fórmula para calcular el tamaño de la muestra:

Se tiene $\mathbf{N}=\mathbf{1 0 0 9}$, para el $95 \%$ de confianza $\mathrm{Z}=1,96$, y como no se tiene los demás valores se tomará y e $=0,05$.

Reemplazando valores de la fórmula se tiene:

$$
n=\frac{N \sigma^{2} Z^{2}}{e^{2}(N-1)+\sigma^{2} Z^{2}}
$$




$$
\begin{gathered}
n=\frac{1009 \cdot 0,5^{2} \cdot 1,96^{2}}{0,1(1009-1)+0,5^{2} \cdot 1,96^{2}} \\
\mathrm{n}=\frac{1009 \cdot 0,25 \cdot 3,8416}{0,01(1008)+0,25 \cdot 3,8416} \\
\boldsymbol{n}=\mathbf{8 8} \text { redondeando } 90
\end{gathered}
$$

Recabado los datos durante las evaluaciones por día, mes, semana se procesaron mediante estadísticas básicas y se elaboraron figuras y tablas. Estas se interpretaron y analizaron.

Las variables de los instrumentos fueron nominales, como la Escala de Likert, el Cuestionario y Prueba Educativa; pero al darle un valor a cada ítem se convirtió en ordinal, motivo a ello, se modificó de cualitativo al enfoque cuantitativo. Por lo tanto, los instrumentos fueron validados por el Juicio de Expertos. También el análisis de fiabilidad con el Alfa de Cronbach. Los análisis estadísticos se realizaron con el programa computacional SPSS (Statistical Package for Social Sciencies) en su versión 22 en español. Asimismo, se utilizó para la sistematización de datos el paquete de Microsoft Office, específicamente, Microsoft Excel. Para el análisis de los datos se utilizó tanto la estadística descriptiva e inferencial y para la aplicación de la prueba de estadística de contraste de U de MannWhitney de prueba no paramétrica se procesó con muestras independientes.

\section{RESULTADOS Y DISCUSIÓN}

El análisis de frecuencia de las puntuaciones alcanzadas después de aplicar los instrumentos en estudiantes, se tabuló y luego se obtuvo un baremo para poder interpretar las tablas, tal como se muestra en la Tabla 1.

En la Tabla 1, se observa que del total de los encuestados (46 alumnos) predomina el nivel medio; es decir más de la mitad representada por el 65,2\% (30) percibe que la aplicación de la lingüística textual ocupa dicho nivel, el 32,6\% (15) en el nivel alto y solo el 2,2\% (1) considera que el nivel es bajo.

Tabla 1. Niveles de aplicación de la lingüística textual.

\begin{tabular}{ccccccc}
\hline & & & Frecuencia & Porcentaje & Porcentaje válido & Porcentaje acumulado \\
\hline Válidos & Bajo & $<=46$ & 1 & 2,2 & 2,2 & 2,2 \\
& Medio & $47-73$ & 30 & 65,2 & 65,2 & 67,4 \\
& Alto & $74-100$ & 15 & 32,6 & 32,6 & 100,0 \\
\hline Total & & $\mathbf{4 6}$ & $\mathbf{1 0 0 , 0}$ & $\mathbf{1 0 0 , 0}$ & \\
\hline
\end{tabular}

Los encuestados hacia el nivel de la aplicación de la lingüística textual son favorables. Siendo el valor que más se repite 2 (favorable). Más del 50\% de los encuestados están por encima de 2.00; es decir tienen una actitud favorable. En promedio los sujetos se ubican en 2,30 (favorable). Asimismo, se desvían con respecto al promedio 0,511 unidades de la escala. Se observó un puntaje mínimo alcanzado de 1 y el máximo de 3 . De acuerdo a los datos obtenidos en las medidas de tendencia central existe una actitud favorable respecto a la aplicación de la lingüística textual según los alumnos encuestados (ver Tabla 2). 
Tabla 2. Niveles de la aplicación de la lingüística textual.

\begin{tabular}{lrr}
\hline \multicolumn{1}{c}{$\mathbf{N}^{\circ}$} & Válidos & $\mathbf{4 6}$ \\
\hline Media & 2,30 \\
Mediana & 2.00 \\
Moda & 2 \\
Desviación estándar & 0,511 \\
Mínimo & 1 \\
Máximo & 3 \\
\hline
\end{tabular}

En base a la Tabla 3, sobre el nivel del grupo experimental (antes) en comprensión lectora y niveles de logro se tiene los siguientes datos: el 93.49\% (43) para la comprensión lectora; el 91,31 $\%(42)$ para los niveles de logros, los alumnos han obtenido notas entre 06 y10 puntos (ambos), esto indica que se encuentran dentro de la valoración deficiente; el 2,7\% (1) para la comprensión lectora; el 2,7 \% (1) para los niveles de logros, los alumnos han obtenido notas entre 00 y 05 puntos (ambos), esto indica que se encuentran dentro de la valoración muy deficiente; el 2,7 \% (1) para la comprensión lectora; el 4,35\% (2) para los niveles de logros, los alumnos han obtenido notas entre 11 y 12 puntos (ambos), esto indica que se encuentran dentro de la valoración regular; el 2,7 \% (1) para la comprensión lectora; el 1,27 \% (1) para los niveles de logros, los alumnos han obtenido notas entre 13 y 16 puntos (ambos),esto indica que se encuentran dentro de la valoración bueno; debido a que los alumnos de alguna manera están informados sobre los temas referentes a la "lingüística textual" antes de empezar con el tratamiento.

Tabla 3. Nivel de grupo experimental (antes).

\begin{tabular}{lccccc}
\hline & \multirow{2}{*}{ Intervalo } & \multicolumn{2}{c}{ Comprensión lectora } & \multicolumn{2}{c}{ Niveles de logros académicos } \\
& & $\mathbf{f i}$ & $\mathbf{f i} \%$ & $\mathbf{f i}$ & $\mathbf{f} \%$ \\
\hline Muy deficiente & $00-05$ & 1 & 2.17 & 1 & 2.17 \\
Deficiente & $06-10$ & 43 & 93.49 & 42 & 91.31 \\
Regular & $11-12$ & 1 & 2.17 & 2 & 4.35 \\
Bueno & $13-16$ & 1 & 2.17 & 1 & 2.17 \\
Muy bueno & $17-20$ & - & - & - & - \\
\hline Total & & $\mathbf{4 6}$ & $\mathbf{1 0 0 , 0}$ & $\mathbf{4 6}$ & $\mathbf{1 0 0 , 0}$ \\
\hline
\end{tabular}

En base a la Tabla 4, sobre el nivel del grupo experimental (antes) en comprensión lectora y niveles de logro se tiene los siguientes datos: el 2,17\% (1) para la comprensión lectora; el 41,30\% (19) para los niveles de logros, los alumnos han obtenido notas entre 11 y 12 puntos (ambos), esto indica que se encuentran dentro de la valoración muy regular; el 86,96 \% (40) para la comprensión lectora ; el 58,70 \% (27) para los niveles de logros, los alumnos han obtenido notas entre 13 y 16 puntos (ambos), esto indica que se encuentran dentro de la valoración Bueno; el 10,87 \% (5) parala comprensión lectora, los alumnos han obtenido notas entre $17 \mathrm{y}$ 20 puntos (ambos),esto indica que se encuentran dentro de la valoración muy bueno; debido a que los alumnos de alguna manera están informados sobre los temas referentes a la "lingüística textual" antes de empezar con el tratamiento. 
Tabla 4. Nivel de grupo experimental (después).

\begin{tabular}{lccccc}
\hline & \multirow{2}{*}{ Intervalo } & \multicolumn{2}{c}{ Comprensión lectora } & \multicolumn{2}{c}{ Niveles de logros académicos } \\
& & $\mathbf{f i}$ & $\mathbf{f i} \%$ & $\mathbf{f i}$ & $\mathbf{f} \%$ \\
\hline Muy deficiente & $00-05$ & - & - & - & - \\
Deficiente & $06-10$ & - & - & - & - \\
Regular & $11-12$ & 1 & 2,17 & 19 & 41,30 \\
Bueno & $13-16$ & 40 & 86,96 & 27 & 58,70 \\
Muy bueno & $17-20$ & 5 & 10,87 & - & - \\
\hline Total & & $\mathbf{4 6}$ & $\mathbf{1 0 0 , 0}$ & $\mathbf{4 6}$ & $\mathbf{1 0 0 , 0}$ \\
\hline
\end{tabular}

Antes de realizar la prueba de normalidad respectiva primero se determinó, si hay una distribución normal de los datos (estadística paramétrica) o no; es decir una libre distribución (estadística no paramétrica). Para tal efecto se utilizó la prueba de normalidad de Kolmogorov Smirnov ( $\mathrm{n}>50)$ (ver Tabla 5).

La prueba de normalidad muestra que en ambos grupos las variables no se distribuyen según una ley normal; ya que la asintótica de ambos grupos es 0,000; es decir para el grupo control, está por debajo del nivel de significación alfa prefijado $(0,05)$; por lo que, para el grupo experimental es 0.013 , está por debajo del nivel de significación alfa prefijada $(0,05)$, lo cual significa que para el análisis de la relación entre estos dos grupos se optó por pruebas no paramétricas. Por lo tanto, los datos no provienen de poblaciones normales, ya que presentan un porcentaje menor al 5\%.

Tabla 5. Prueba de normalidad.

\begin{tabular}{clcccccc}
\hline \multirow{2}{*}{$\begin{array}{c}\text { Niveles } \\
\text { de logro }\end{array}$} & & \multicolumn{3}{c}{ Kolmogorov-Smirnova } & \multicolumn{3}{c}{ Shapiro-Wilk } \\
académico & Control & Estadístico & g.l. & Asintótica & Estadístico & gl & Sig. \\
& Experimental & 0,306 & 46 & 0,000 & 0,881 & 46 & 0,000 \\
\hline
\end{tabular}

a. Corrección de la significación de Lilliefors.

Como la prueba U de Mann-Whitney vale 1435,500 y el p-valor es 0,000 (sig. bilateral) menor que 0.05 , entonces se rechaza la hipótesis nula y se acepta la hipótesis alterna, por lo tanto, la aplicación de la lingüística textual tiene efecto significativo en los niveles de logro académicos son estadísticamente diferentes al nivel de significación alfa $=0,05$. Los resultados demuestran que en los niveles de logro académicos es ligeramente mayor que los niveles de logro, la diferencia puntual de estas medias es 2,17 puntos. se confirma que, en la prueba de Rango, si existen diferencias significativas (ver Tabla 6). 
Tabla 6. Estadísticos de contraste.

\begin{tabular}{lc}
\hline \multicolumn{1}{c}{ Estadísticos de contrastea } & Niveles de logro académico \\
\hline U de Mann-Whitney & 354,500 \\
W de Wilcoxon & 1435,500 \\
Z & $-5,655$ \\
Sig. asintót. (bilateral) & 0,000 \\
\hline
\end{tabular}

Regla de decisión

- Si p-valor < 0.05 se sitúa en la región crítica o región de rechazo, entonces rechaza la H0 "existe diferencia significativa".

- Si p-valor $>0.05$ se encuentra dentro de la región de aceptación, entonces asume la H0 "no existe diferencia significativa".

Los resultados de este estudio muestran que los sujetos del grupo experimental, en el análisis estadístico de la hipótesis central o principal permite rechazar la (Ho) y aceptar la hipótesis alterna (Ha), al confirmarse de que existe diferencias significativas después de la aplicación de la lingüística textual en la comprensión lectora y los niveles de logro académico de los estudiantes en las habilidades cognitivas, lingüísticas, discurso pragmático, en el acto del habla, en el signo complejo o semiótico, proceso multifactorial, activo e interacción e interdisciplinario. Por lo tanto, se ha demostrado un nivel de confianza del 95\%, siendo la significación asintótica (bilateral) 0,000, la diferencia puntual de estas medias es 1,72 puntos y la prueba de normalidad muestra que en ambos grupos las variables, no se distribuyen según una ley normal, ya que la asintótica (bilateral) de ambos grupos es 0,013; es decir, para la comprensión lectora está por debajo del nivel de significación alfa prefijado $(0,05)$.

\section{Discusión}

Al respecto, Van Dijk (1980) confirma los resultados al explicar que la lingüística de la gramática tiene un método concreto; es decir, en el ámbito de la ciencia del lenguaje, este término se utiliza para cualquier tipo de estudio relacionado con el texto. Por otro lado, Spillner (1974) expresa que la lingüística textual explora lo siguiente: Acceso a organizar las ideas, conjuntos de ideas, la emisión de juicio y la interacción comunicativa. Por lo consiguiente, Antos (1997) identifica las siguientes concepciones del texto como: La oración compleja, el signo complejo, acto del habla, el discurso y verbalizaciones de operaciones y procesos cognitivos.

Así mismo, Puente (1994) menciona en los modelos interactivos se postula que ocurre tanto el procesamiento ascendente como el descendente, siempre predomina uno, dependiendo del dominio lingüístico del lector, de su conocimiento previo del mundo y de su habilidad lectora y del tema y la dificultad del texto. Como podernos ver, los esquemas cognitivos del lector desempeñan un papel importante según estos modelos, porque son el constructo semántico responsable de la asimilación de la información nueva. El enfoque interactivo ha logrado mucha aceptación en los últimos años, por lo cual hoy está bastante aceptado que el procesamiento de un texto es un proceso interactivo y que el significado no está en el texto, sino que es el lector quien lo construye.

Según Ortiz, (2006) expresa el camino hacia el cumplimiento del estándar que refleja los propósitos, metas y aspiraciones a alcanzar por el estudiante, 
desde el punto de vista afectivo (sentir), cognitivo (pensar) e instrumental (actuar). El indicador de desempeño también es un logro, pero es un nivel más inferior, es un logro más específico y detallado. De otro lado, se observa en los resultados de la (He) (hipótesis especifica), en donde la Hipótesis alterna se acepta, debido a que existe diferencia significativa entre antes y después de la aplicación de la lingüística textual en la comprensión lectora de los estudiantes. Así mismo, se manifiesta una diferencia estadísticamente significativa entre el pretest y postest, la diferencia puntual de estas medias es 5,76 puntos. Por lo consiguiente, la prueba de normalidad muestra que en ambos grupos las variables, no se distribuyen según una ley normal, ya que la sig. asintótica (bilateral) de ambos grupos es 0,013 , es decir, para la comprensión lectora está por debajo del nivel de significación alfa prefijado $(0,05)$.

También plantea Van Dijk (1980), que la lingüística textual va mucho más allá de la ampliación de la unidad de análisis del texto como: La producción y la comprensión del texto, el estudio interdisciplinario del lenguaje, así mismo se introduce varias categorías nuevas en el estudio del discurso. Por su parte, Puente, (1994) ha contrastado que mediante la lectura accede a un vasto mundo de informaciones, las cuales son necesarias para estar al día y de esta manera responder a las exigencias sociales e intelectuales. En el ámbito escolar, la lectura es un instrumento fundamental para el aprendizaje posterior, de tal manera que los problemas de lectura producirán problemas porque obstaculizan el aprovechamiento escolar. La lectura implica una amplia variedad de procesos y destrezas cognitivas: atención, percepción, memoria, etc.
El conocimiento de estos procesos facilitará la comprensión de la información y las formas para mejorar la lectura.

Existe otro grupo de modelos, que enfatizan la comprensión tomando en cuenta unidades mayores que la oración (macroprocesos). En otras palabras, estos modelos se centran en la estructura del texto (Kintsch y Van Dijk, 1978; Kintsch, 1988, citados por Puente, 1994). Estos autores han presentado un modelo muy amplio que trata de explicar los procesos involucrados en la comprensión de un texto. De acuerdo con este modelo, la comprensión de un texto no se reduce a extraer el significado de las oraciones o enunciados, puesto que el texto es algo más que la suma de enunciados que lo forman. Al procesar un texto, el lector debe integrar sus enunciados y hacer inferencias de acuerdo a sus esquemas cognitivos y su conocimiento del mundo; todo ello le permitió interpretar el texto que lee.

De otro lado, se observa en los resultados de la He (hipótesis especifica), en donde la Hipótesis alterna se acepta, debido a que aplicación de la lingüística textual tiene efecto significativo en la comprensión lectora de los estudiantes. En que se manifiesta una diferencia estadísticamente significativa entre el pretest y postest, la diferencia puntual de estas medias son 1,61 puntos. La prueba de normalidad muestra que en ambos grupos las variables, no se distribuyen según una ley normal, ya que la asintótica (bilateral) de ambos grupos es 0,000; es decir, para los niveles de logro académicos está por encima del nivel de significación alfa prefijado (0,05). En donde afirma Solé (1986) que la lectura como un proceso secuencial y jerárquico. El procesamiento es unidireccional, con fuerte incidencia de un nivel sobre el siguiente. Esto se 
debe a que estos modelos colocan el significado en el texto, con lo cual el papel del lector se reduce casi al de simple decodificador de un significado ya dado y al cual tiene muy poco que aportar.

Según Camina (1996) demostró en su investigación que le da muy poca importancia a la decodificación de letras y palabras, por lo cual parece ser más aplicable al lector experto y no así al novato. Además, según Puente (1994), han demostrado que los buenos lectores emplean las siguientes estrategias para comprender lo que leen: Aclarar los propósitos de la lectura, esto es, entender cuáles son las tareas implícitas y explícitas que se exigen; identificar los aspectos importantes del mensaje; centrar la atención en la información relevante más que en la trivial; controlar las actividades para determinar si se está comprendiendo o no; hacerse preguntas a sí mismo para dominar si los objetivos se están logrando; tomar acciones correctivas cuando se detectan fallas en la comprensión.

Aclarando según Beaugrande, y Dressler (1997) plantea en su investigación de la lingüística del texto se manifiesta diferencia significativa en los niveles de logro académico en el acto del habla, el sentido, la referencia, contexto y la semántica lógica. Definió el estilo cognitivo como el modo habitual o típico de una persona para resolver problemas, pensar, percibir y recordar en la menciona (Tenant, 1988).

Según Moreno (1998) ha comprobado en su resultado que el logro académico es como una categoría que intenta compendiar todo aquello que un estudiante alcanza como resultado directo. En marcado las siguientes dimensiones de logro son: La construcción de conocimientos, el desarrollo de habilidades, la formación de hábitos y actitudes, y la internalización de valores, entre otras. Como se desprende de su definición, el logro académico es una variable de naturaleza individual.

\section{CONCLUSIONES}

Se acepta la hipótesis alterna (HG) y se rechaza la (Ho) al confirmarse que existe diferencia significativa después de la aplicación de la lingüística textual; es decir, la media de la comprensión lectora y los niveles de logro son estadísticamente diferentes al nivel de significación alfa $=0,05$. Los resultados demuestran que el nivel de comprensión lectora es ligeramente mayor que los niveles de logro, la diferencia puntual de estas medias es 1,72 puntos.

Se acepta la hipótesis alterna $(\mathrm{He})_{1}$ y se rechaza la hipótesis nula (Ho) al confirmarse que existe diferencia significativa entre antes y después de la aplicación de la lingüística textual en la comprensión lectora de los estudiantes. Los resultados demuestran que si existe diferencia significativa entre antes y después de la aplicación de la lingüística textual en la comprensión lectora en los alumnos. Por lo tanto, es mayor después de la aplicación de la lingüística textual es 5,75 puntos.

Por lo tanto, la hipótesis alterna $(\mathrm{He})_{2}$ y se rechaza la hipótesis nula (Ho) al confirmarse que la aplicación de la lingüística textual tiene efecto significativo en la comprensión lectora de los estudiantes; por lo tanto, son estadísticamente diferentes al nivel de significación alfa $=0,05$. Los resultados demuestran que el nivel de comprensión lectora en el grupo experimental es ligeramente mayor que en el grupo control la diferencia puntual de estas medias es 1,61 puntos. 


\section{REFERENCIAS}

Albarrán, M. (2015). La lingüística textual en los trabajos de investigación producidos por alumnado universitario: Enseñanza en las planificaciones de aula y los libros de texto. Revista Electrónica Educare (Educare Electronic Journal) EISSN: 1409-4258 Vol. 19(1) enero-abril, 2015: 389-406/doi: http://dx.doi. org/10.15359/ree.19-1.21

Antos, G. (1997). Textos como Constitución-formas de conocimiento: El futuro de la lingüística del texto. México Paidós, pp. 43-63

Beaugrande, R. A., y Dressler, W. U. (1997). Introducción a la Lingüística del texto. $1^{\text {a }}$ edición en español. Editorial Ariel, SA Barcelona España

Camina, M. T. (1996). Experiencias de recuperación de las dificultades en lectura en el $3^{\circ}$ de E.G.B. Tesis no publicada. Universidad Complutense de Madrid, Madrid

Cavalcante, M. (2016). Abordagens da argumentação nos estudos de Linguística Textual. ReVEL, edição especial vol. 14, n. 12, 2016. [www.revel. inf.br]- ISSN 1678-8931/ [Consultado 12 de diciembre 2019]

Espinoza H., Carrillo L., Valentin G., Ramos J. y Acero L (2021). Estrategias pedagógicas para desarrollar aprendizajes significativos y mejorar las actitudes hacia la matemática. Horizontes. Revista de Investigación en Ciencias de la Educación. Octubre- diciembre 2021-Volumen 5 / No. 21ISSN: 2616-7964ISSN-L: 26167964pp. 1375 - 1387/ Https: //doi.org/10.33996/ revistahorizontes.v5i21.282

Gómez, M. (2006). Introducción a la metodología de la investigación científica. Editorial Brujas, Argentina

Gonzales, H. (2010). Propuesta de lectura desde la lingüística textual. Revista Pueblo Continental Vol. 21 (1). pp. 125-129. ISSN- 1991-5837http://journal.upao.edu.pe/PuebloContinente/ article/viewFile/519/481/ [Consultado 13 de diciembre 2019]

Kintsch, Walter (1988) "The Role of Knowledge in Discourse Comprehension: A constructionintegration model", Psychological Review, vol. 95, núm. 2, pp. 163-183
Kintsch, W., y Van Dijk, T. A. (1978). Toward a model of text comprehension and production. Psychological review, 85(5), 363

Mamani, H. et al (2021). Implicancias de la neuroeducación y desempeño docente: desde la perspectiva del estudiantado. Horizontes. Revista de Investigación en Ciencias de la Educación/ Volumen 5 / No. 20, Edición Extraordinaria septiembre 2021ISSN: 2616-7964ISSN-L: 26167964pp. 1273 - 1287/ Https://doi.org/10.33996/ revistahorizontes.v5i20.276

Moreno, A. (1998). El desarrollo de habilidades como objeto educativo. Una aproximación conceptual. Educar. Nueva Era. Julio - setiembre.

Muñoz, A. y Ocoña, M. (2017). Uso de estrategias metacognitivas para la comprensión textual. Cuadernos de Lingüística Hispánica, (29), 223244- http://dx.doi.org/10.19053/0121053X. n29.2017.5865 [Consultado 12 de diciembre 2019]

Ortiz, A. (2006). Evaluación formativa. Barranquilla: Editorial Cepedid

PISA, (2009). Programa Internacional para la Evaluación de Estudiantes o Informe. Mejores políticas para una vida mejor. [Consultado 28 de enero 2021]. Página web https://n9.cl/xaxre

Puente, A. (1994). Comprensión de la lectura y metacognición. Madrid: CEPE. Págs. 107- 127

Sánchez H. y Reyes C. (1996). Metodología y diseño de la investigación científica. Lima: Editorial Mantaro. $30 \mathrm{p}$

Santos, A. (2020). Rendimiento escolar en la asignatura de Lenguaje y Comunicación en el nivel Secundario de Bolivia. Horizontes. Revista de Investigación en Ciencias de la Educación. Octubre -Diciembre2020Volumen 4 / No. 16ISSN: 2616 -7964ISSN-L: 2616 -7964pp. 590 -604/ Https://doi.org/10.33996/ revistahorizontes.v4i16.139

Silva, M. y De Paiva, M. (2017). A linguística textual e a construção do texto: um estudo sobre os fatores de textualidade. Revista Digital dos Programas de Pós-Graduação do Departamento de Letras e Artes da UEFS Feira de Santana, v. 18, n. 2, p. 26-44, mayo-agosto 2017/ http:// dx.doi.org/10.13102/cl.v18i2.1866 
Spillner, B. (1974). Linguistik und Literaturwissenschaft: Stilforschung, Rhetorik, Textlinguistik. W. Kohlhammer

Solé, I. (1986). Desenyament de la comprensión lectora: un punto de vista interactiun. Tesis doctoral. Facultad de psicología. Universidad de Barcelona. file://C:/Users/Usuario/Downloads/ Dialnet-LasPosibilidadesDeUnModeloTeoricoP araLaEnsenanzaDe-749227.pdf

Souza, S. (2017). El concepto de intertextualidad e interdiscursividad en el lenguaje textual y el análisis del discurso: perspectivas. Lingu@ Nostr@ - Revista Virtual de Estudios de Gramática e Lingüística do Curso de Letras da
Facultad de Tecnología IPUC - FATIPUC-ISSN 2317-2320/Língu@ Nostr@, Canoas, v. 5, n. 1, p. 97 - 111, jan.-jun. 2017 | / [Consultado 12 de diciembre 2019]

Tenant, M. (1988). Psicología y Aprendizaje de Adultos. Nueva York: Routledge, 182 p. https://journals.sagepub.com/doi/ abs/10.1177/074171369204200311

Van Dijk, T (1980) Estructuras y funciones del discurso. Una introducción interdisciplinaria a la lingüística del texto y a los estudios del discurso. México: Siglo XXI 